\title{
On faint companions in the close environment of star-forming dwarf galaxies
}

\section{Possible external star formation triggers?}

\author{
K. G. Noeske ${ }^{1, \star}$, J. Iglesias-Páramo ${ }^{2}$, J. M. Vílchez ${ }^{3}$, P. Papaderos ${ }^{1}$, and K. J. Fricke ${ }^{1}$ \\ 1 Universitäts-Sternwarte Göttingen, 37083 Göttingen, Germany \\ 2 Instituto de Astrofísica de Canarias, 38200 La Laguna, S/C de Tenerife, Spain \\ 3 Instituto de Astrofísica de Andalucía (CSIC), 18080 Granada, Spain
}

Received 25 September 2000 / Accepted 12 March 2001

\begin{abstract}
We have searched for companion galaxies in the close environment of 98 star-forming dwarf galaxies (SFDGs) from field and low density environments, using the NASA Extragalactic Database. Most of the companions are dwarf galaxies which due to observational selection effects were previously disregarded in environmental studies of SFDGs. A subsample at low redshift, $c z<2000 \mathrm{~km} \mathrm{~s}^{-1}$, was chosen to partially eliminate the observational bias against distant dwarf companions. We find companion candidates for approximately $30 \%$ of the objects within a projected linear separation $s_{\mathrm{p}}<100 \mathrm{kpc}$ and a redshift difference $\Delta c z<500 \mathrm{~km} \mathrm{~s}^{-1}$. The limited completeness of the available data sets, together with the non-negligible frequency of $\mathrm{H}$ I clouds in the vicinity of SFDGs indicated by recent radio surveys, suggest that a considerably larger fraction of these galaxies may be accompanied by low-mass systems. This casts doubt on the hypothesis that the majority of them can be considered truly isolated. The velocity differences between companion candidates and sample SFDGs amount typically to $\lesssim 250 \mathrm{~km} \mathrm{~s}^{-1}$, and show a rising distribution towards lower $\Delta c z$. This is similarly found for dwarf satellites of spiral galaxies, suggesting a physical association between the companion candidates and the sample SFDGs. SFDGs with a close companion do not show significant differences in their $\mathrm{H} \beta$ equivalent widths and $B-V$ colours as compared to isolated ones. However, the available data do not allow us to rule out that interactions with close dwarf companions can influence the star formation activity in SFDGs.
\end{abstract}

Key words. galaxies: dwarf - galaxies: compact - galaxies: evolution - galaxies: interaction

\section{Introduction}

Dwarf galaxies are considered important contributors to the baryonic mass of the Universe and to the star formation rate (SFR) density at higher redshifts (see. e.g. Guzmán et al. 1998). Some of them may even be similar to low-mass building blocks of normal galaxies in a bottom-up cosmological model. A better understanding of the processes that drive their evolution is therefore central to cosmological studies, and to the understanding of galaxy formation and evolution.

Starbursts, i.e. brief episodes of strongly enhanced star formation (SF) activity, are thought to occur frequently during the lifetime of a gas-rich dwarf galaxy. A number of internal processes has been put forward to explain their origin and transient nature, such as Stochastic

\footnotetext{
Send offprint requests to: K. Noeske,

e-mail: knoeske@uni-sw.gwdg.de

* Summer research student 1998, Instituto de Astrofísica de Canarias.
}

Self-Propagating Star Formation (Gerola et al. 1980; see Thuan 1991) or a cyclic process of gas infall onto and expulsion from an older stellar host (e.g. Davies \& Phillips 1988; Papaderos et al. 1996).

Alternatively, environmental influences have been suggested to induce starburst activity in star-forming dwarf galaxies (SFDGs; see Sect. 2). Based on the hypothesis that dwarf galaxies are affected in a similar way to luminous disk galaxies (e.g. Kennicutt et al. 1987), mainly tidal interactions with stellar or gaseous companions have been treated by former studies.

Information on the presence and frequency of such external perturbors has been assembled from environmental studies of emission line galaxies (ELGs), performed mainly in the context of large scale structure formation and biased formation and evolution of galaxies in different environments. ELGs were found to smoothly follow the structures delineated by luminous galaxies, although they are more fuzzily distributed (Rosenberg et al. 1994), generally populating lower-density environments (Salzer 1989; 
Telles \& Terlevich 1995, hereafter TT95), and are less strongly clustered than less active luminous galaxies (Loveday et al. 1999; Telles \& Maddox 1999; Lee et al. 2000). As for SFDGs, they were found to be concentrated towards void boundaries (Lindner et al. 1996, hereafter L96), with a fraction $(\approx 20 \%)$ of them inside voids (Pustilnik et al. 1995), arranged in loose groups which do not contain any bright galaxies (L96). Interactions with luminous companions were therefore considered too rare to be the generic triggering agent of starbursts in SFDGs, given that luminous galaxies are rare in the close neighbourhood of SFDGs.

Whether interactions with companion galaxies can significantly influence SF activity and evolution of SFDGs has been investigated in earlier work (Campos-Aguilar et al. 1991, 1993; TT95). The spectrophotometric properties of the studied objects showed no significant dependence on the presence or absence of a companion galaxy, and both SF activity and metallicity seemed unrelated to distance or absolute magnitude of putative companions. Campos-Aguilar et al. chose their samples from the spectrophotometric catalogue of HII galaxies (SCHG, Terlevich et al. 1991), which mostly covers redshifts $c z \gtrsim 2000 \mathrm{kms}^{-1}$. Taking into account the magnitude cutoff of the CfA catalogue used for the companion search (13.5 mag), these studies were practically restricted to luminous companions ( $M \lesssim-18 \mathrm{mag}$ ), typically not particularly close to SFDGs. TT95, on their part, explicitly focus on the possible influence of luminous companions $(M<-19 \mathrm{mag})$. Also, the opposite conclusion by Grogin \& Geller (2000), that ELGs in low-density environments show enhanced SF activity in the presence of a companion, is mostly drawn from studies of luminous ELGs and companions.

Observational evidence of a different kind points towards a correspondence between the membership of a SFDG in a given environment and its spectrophotometric properties. A comparative study of SF activity in SFDGs populating different environments by Vílchez $(1995,1997)$ suggests that objects in low density environments have higher SF activity than those located in high density regions. The results of Hashimoto et al. (1998) point in the same direction. Popescu et al. (1999) reported no conspicuous difference in present and past SF activity for ELGs in field and void environments. On the other hand, Vennik et al. (2000) found that the low surface brightness hosts of non-isolated ELGs tend to be more compact than those of isolated ones.

The mixed evidence for an influence of the environment on the SF activity of a SFDG calls for further studies, extending previous ones to larger samples and fainter magnitudes. This seems particularly important in view of the conjecture by L96, who attributed the apparent isolation of a fraction of BCDs to an observational bias against their low-luminosity companions.

Assuming that companions are capable of inducing SF activity in SFDGs, the mechanism and the prime parameters controlling this process remain unclear. Tidal forces are, however, considered to be of major importance. If $D_{\mathrm{c}}$ and $M_{\mathrm{c}}$ denote respectively the distance and mass of a companion, the tidal forces acting on a SFDG scale as

$F_{\mathrm{tid}} \propto M_{\mathrm{c}} \times D_{\mathrm{c}}^{-3}$

so that a nearby low-mass, i.e. faint, perturbor can affect a SFDG to the degree a more distant giant galaxy does (cf. Campos-Aguilar et al. 1991). Observational data (Wilcots et al. 1996) and numerical models (Hensler et al. 1999; Pilyugin 2000) further suggest that, in addition to tidal interactions, low-mass stellar or gaseous companions may trigger and fuel starbursts by infalling onto a SFDG.

Although Telles \& Maddox (1999), from an analysis of APM catalogues, found no excess of dwarf companions for $\mathrm{H}$ II galaxies down to $\approx-14.5 \mathrm{~B}$ mag and $\sim 10^{8} \mathrm{M}_{\odot}$, numerous results point to the presence of objects of even lower masses and luminosities. Non-catalogued objects of low optical luminosities and surface brightnesses were found for most one-armed magellanic irregulars (Odewahn 1994), and around many BCDs (Pustilnik et al. 1997; Walter et al. 1997; Doublier et al. 1999; Méndez \& Esteban 1999).

Likewise, H I observations of the environment of magellanic irregulars (Wilcots et al. 1996) and H II galaxies (Taylor et al. 1994, 1995, 1996) revealed H I companions for the majority of them, down to intergalactic H I clouds with masses of $\sim 10^{7} M_{\odot}$ for some of which no optical counterpart was found.

The presence of still undetected objects of low optical and $\mathrm{H}$ I luminosity appears also likely, keeping in mind that the LF steepens towards the low-luminosity end for SFDGs (Loveday et al. 1999), and in view of e.g. the high space density and the masses of local Ly $\alpha$ absorbers (Shull et al. 1996). Grogin \& Geller (2000) found for luminous ELGs that the frequency of companions at low redshift differences is widely independent of the local galaxy density. Therefore, faint companions may not be rare even in regions of low density of luminous galaxies.

The aim of this study is to investigate the incidence of close optical companions of SFDGs and whether such close companions strongly affect a SFDG's SF activity. Particular attention is attached to faint close companions, the presence and influence of which was generally not assessed in previous work. In order to address the problem of observational bias against low-luminosity sources, we investigate separately a subset of nearby SFDGs with recession velocities $<2000 \mathrm{~km} \mathrm{~s}^{-1}$. For a sample of SFDGs, we compiled photometric and spectroscopic properties at different wavelengths and corrected distance dependent observables for the Virgocentric infall.

The paper is organized as follows: in Sect. 2 we describe our galaxy sample and the data processing. In Sect. 3, we analyse our data sets by applying different statistical methods and list the results, which are further discussed in Sect. 4. In Sect. 5, we summarize our work and conclusions. We assume $H_{0}=75 \mathrm{~km} \mathrm{~s}^{-1} \mathrm{Mpc}^{-1}$ throughout. 


\section{The data}

\subsection{Sample selection}

Various criteria were applied to select samples of dwarf galaxies with current or recent strong $\mathrm{SF}$, which resulted in catalogues of Blue Compact Dwarf Galaxies (BCDs, e.g. Thuan \& Martin 1981) and H II galaxies (e.g. SCHG, Terlevich et al. 1991). Despite the different classification schemes, it has been subsequently shown that the underlying stellar hosts of starbursting dwarf galaxies have generally a compact structure (Papaderos et al. 1996; Marlowe et al. 1997; Salzer \& Norton 1998). Also comparative studies of dwarf H II galaxies, BCDs and dwarf amorphous galaxies with ongoing SF showed them to widely share the colours and structural properties of their host galaxies, relative starburst luminosities and $E W(\mathrm{H} \alpha)$, suggesting that they basically form one and the same class of extragalactic objects (Papaderos et al. 1996; Telles et al. 1997; Marlowe et al. 1999). We will therefore unify both BCDs and H II galaxies using the term "star-forming dwarf galaxies" (SFDGs), following Vílchez $(1995,1997)$.

In order to study a sufficiently large SFDG sample for which comparable observational data are available, we decided to use lists IV and V of the sample of ELGs from the University of Michigan (UM) Survey (McAlpine et al. 1977a, 1977b, 1977c; McAlpine \& Lewis 1978; McAlpine \& Williams 1981), systematically investigated in Salzer et al. (1989a, 1989b) by means of spectroscopy and CCD imaging in $B$ and $V$. Among the different types of ELGs, all objects classified as $\mathrm{H}$ II galaxies were selected; the magnitude limit of $M_{B}>-18 \mathrm{mag}$, commonly adopted to select dwarf galaxies, was applied after the distance correction (Sect. 2.3) had been performed. More than $80 \%$ of these UM galaxies are located at redshifts $c z>2000 \mathrm{~km} \mathrm{~s}^{-1}$.

Our sample further includes SFDGs studied by Cairós and Noeske (cf. Cairós \& Vílchez 1998; Cairós et al. 2000; Noeske et al. 1998a; 1998b; Noeske 1999). These are, for the most part, nearby objects $\left(c z<2000 \mathrm{~km} \mathrm{~s}^{-1}\right)$ which allow an environmental search that is less biased against faint objects than the quite distant UM sample. None of our sample galaxies are members of galaxy clusters. Those which are known or likely members of groups (cf. NED and Garcia 1993) were rejected (Mkn 35, Mkn 71 and Mkn 527). Only 3 galaxies, UM 454, UM 455 and UM 513 , have been reported to lie inside voids (Salzer 1989). The resulting sample consists of 98 dwarf galaxies in typical field- and low density environments and is listed in Table 3 .

\subsection{Search for companion objects}

Our companion search catalogue was the NASA Extragalactic Database $^{1}$ (NED).

\footnotetext{
${ }^{1}$ The NASA/IPAC Extragalactic Database (NED) is operated by the Jet Propulsion Laboratory, California Institute of Technology, under contract with the National Aeronautics and Space Administration.
}

Most of our objects have declinations $\delta>-3^{\circ} 30^{\prime}$, i.e. lie within the sky region covered by the CGCG (Zwicky 1961), which is included into the NED (cf. TT95). We can therefore attribute the completeness limit of the CGCG, $m_{B}^{\lim } \approx m_{\mathrm{phot}}^{\lim } \approx 15.5 \mathrm{mag}$, to our companion search. As the NED includes numerous other literature sources, considerably fainter objects should partly be detectable, which makes NED a suitable catalogue to find as many known faint sources as possible. The non-uniform completeness of this database does, on the other hand, not constitute a drawback since a meaningful extrapolation beyond even a well-defined completeness limit is at present not possible (see Sect. 4.1).

In an initial selection procedure, we considered any type of extragalactic source a possible companion. Statistical analyses of spatial companion frequencies from excess source densities around SFDGs have been previously done (Telles \& Maddox 1999). We hence restricted ourselves to sources for which redshift data was available. Thereby some companions are missed, but information on individual companions' properties such as luminosity etc. can be analyzed.

Any object within a projected separation $s_{\mathrm{p}} \leq 0.1 \mathrm{Mpc}$ from our sample galaxies was included into a tentative list of companions. The corresponding angular search radius was calculated from the redshift $c z$ given in the NED, assuming a pure Hubble flow. We allowed, in the first place, a maximum separation in velocity space of $\Delta c z \leq 2000 \mathrm{~km} \mathrm{~s}^{-1}$ between sample object and companion. The final selection criterion below which we finally considered an object a companion was, however, $\Delta c z \leq 500 \mathrm{~km} \mathrm{~s}^{-1}$ (cf. Sect. 3.1.1).

\subsection{Distances and correction for Virgo Cluster infall}

The choice of a less distant subsample of SFDGs with $c z<2000 \mathrm{~km} \mathrm{~s}^{-1}$ does not justify the assumption of a pure Hubble flow throughout to determine galaxy distances, but requires a correction for the peculiar velocity field in the environment of the Virgo Cluster (VC). For this purpose, we adopted the distances listed in the Nearby Galaxies Catalog (Tully 1988, hereafter T88). These are based on a Virgocentric infall model, which uses redshift - independent distance determinations, morphology and neighbourhood considerations to treat those galaxies affected by the triple value problem (see Tully \& Shaya 1984).

For all objects not listed in T88, the distance was directly calculated from their redshift given in the NED. Galaxies within the redshift range covered by T88, $c z<3000 \mathrm{~km} \mathrm{~s}^{-1}$, which are not listed in the catalogue, were found to be at least 1.5 mag fainter than the dwarf - non dwarf separation limit of $M_{B}>-18 \mathrm{mag}$, i.e. can be considered dwarfs despite their possibly larger distance insecurities. For galaxies outside the redshift range of $\mathrm{T} 88$, the deviation of the true distance from the one obtained assuming a pure Hubble Flow can be estimated 
from Kraan-Korteweg (1986, cf. their Fig. 3b) to $\lesssim 30 \%$ due to VC infall effects. A comparable deviation was obtained by Marinoni et al. (1998, cf. their Fig. 14) who have adopted in their study a multiattractor model. The abovementioned dispersion translates in the worst case into an uncertainty of $\$ 0.6 \mathrm{mag}$ in the distance modulus which may then slightly affect the luminosity-based distinction between dwarf and luminous galaxies. This does not affect the distance-independent quantities $B-V$ and $E W(\mathrm{H} \beta)$ involved in our study.

For the companion candidates, we assumed the same distances as for the sample galaxies around which they were found. Given that the peculiar velocity field in the $\mathrm{VC}$ vicinity is not expected to change dramatically on scales of few tens of kpc, and that the companions show signs of physical association with the sample galaxies (cf. Sect. 3.1.1), the above assumption appears justified. For these distances, projected separations $s_{\mathrm{p}}$ between sample objects and candidate companions were calculated from their angular separation.

\subsection{Photometric and spectroscopic data}

Aperture photometry in optical broadbands was taken from the NED i.e. listing mostly RC3 data, from Salzer (1989a, 1989b), Cairós et al. (2000), Noeske (1999), and Noeske et al. (1998a, 1998b). H $\beta$ equivalent widths were compiled from French (1980), Thuan \& Martin (1981), the SCHG (Terlevich et al. 1991) and Salzer (1989a, 1989b).

Adopting the distances obtained as described in Sect. 2.3, apparent magnitudes were transformed to absolute values. A correction for galactic foreground dust extinction was applied to all photometric data using the $B$ band extinction given in the NED for each galaxy, and applying the standard reddening curve by Savage \& Mathis (1979).

\section{Results}

\subsection{The close environment}

\subsubsection{Distribution of the companions and companion selection criteria}

It is conceivable that the recession velocity difference, $\Delta c z$, and the projected separation, $s_{\mathrm{p}}$, strongly influence the dynamical response and spectrophotometric evolution of dwarf galaxies in the presence of a companion.

We assumed tidal forces to have significant dynamical influence within the range $\Delta c z<500 \mathrm{~km} \mathrm{~s}^{-1}$ and $s_{\mathrm{p}}<0.1 \mathrm{Mpc}$. These criteria follow the estimates by Campos-Aguilar et al. (1993), and are comparable to those by Pustilnik et al. (2001). They are further compatible with observed values of $s_{\mathrm{p}}$ and $\Delta c z$ for e.g. the BCD II Zw 33 and its companion II Zw $33 \mathrm{~B}$, which are probably bound (Walter et al. 1997) and likely to be influenced by interaction (Méndez et al. 1999). For M51-like close pairs of galaxies, which show signs of perturbation
Table 1. Companion search results for subsamples within different redshift intervals

\begin{tabular}{|c|c|c|c|c|}
\hline $\begin{array}{l}c z^{a} \\
\text { range } \\
(1)\end{array}$ & $\begin{array}{c}\text { sample } \\
\text { size } \\
(2)\end{array}$ & $\begin{array}{c}\text { non } \\
\text { isol. } \\
(3)\end{array}$ & $\begin{array}{c}\text { no. of } \\
\text { comps. } \\
(4)\end{array}$ & $\begin{array}{c}\text { dwarf } \\
\text { comps. } \\
(5)\end{array}$ \\
\hline unconstrained & 98 & 16 & 18 & 15 \\
\hline$c z<2000$ & 42 & 13 & 15 & 14 \\
\hline $2000 \leq c z<4000$ & 12 & 2 & 2 & 1 \\
\hline $4000 \leq c z<6000$ & 20 & 0 & 0 & 0 \\
\hline $6000 \leq c z<8000$ & 15 & 1 & 1 & 0 \\
\hline$c z \geq 8000$ & 9 & 0 & 0 & 0 \\
\hline
\end{tabular}

${ }^{a}$ redshifts are corrected for Virgo infall by multiplying the corrected distances from Sect. 2.3 by $H_{0}\left(75 \mathrm{~km} \mathrm{~s}^{-1} \mathrm{Mpc}^{-1}\right)$.

(1) redshift interval (in $\mathrm{km} \mathrm{s}^{-1}$ ) of respective subsample.

(2) number of sample galaxies in subsample.

(3) number of galaxies in the subsample for which at least one possible companion was found.

(4) total number of possible companions found for the respective subsample.

(5) number of dwarf galaxies among the possible companions.

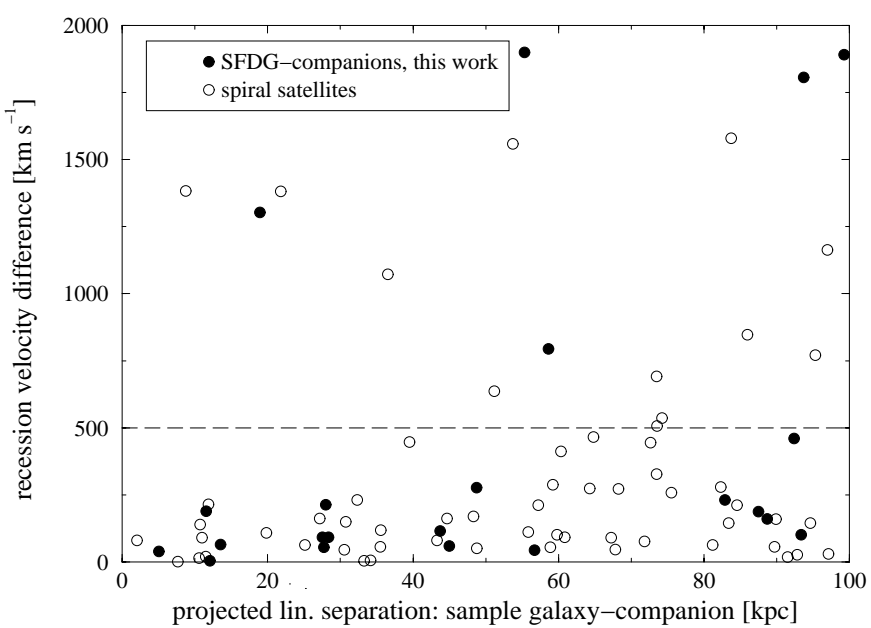

Fig. 1. Recession velocity difference vs. projected linear separation of the SFDGs' putative companions (filled symbols). For comparison, we show by open circles the distribution of all companions (not restricted to dwarf galaxies) we found around field spiral galaxies from the sample of Kennicutt \& Kent (1983). The dashed line represents the maximum recession velocity difference between SFDG and companion we adopt

due to interaction, similar values for $\Delta c z$ are observed (Laurikainen et al. 1998).

The distribution of our SFDG companion candidates on the $\Delta c z$ vs. $s_{p}$ plane (Fig. 1, filled circles) peaks towards small redshift differences. This is also illustrated in Fig. 2, which shows the frequency of the companions to be rising towards smaller $\Delta c z$. If this trend reflects a physical association between sample SFDG and companion (see Sect. 4), then our cutoff, $\Delta c z<500 \mathrm{~km} \mathrm{~s}^{-1}$, should mainly select true companions, i.e. objects likely to act as perturbors repeatedly or on longer timescales, rather than randomly passing interlopers. 


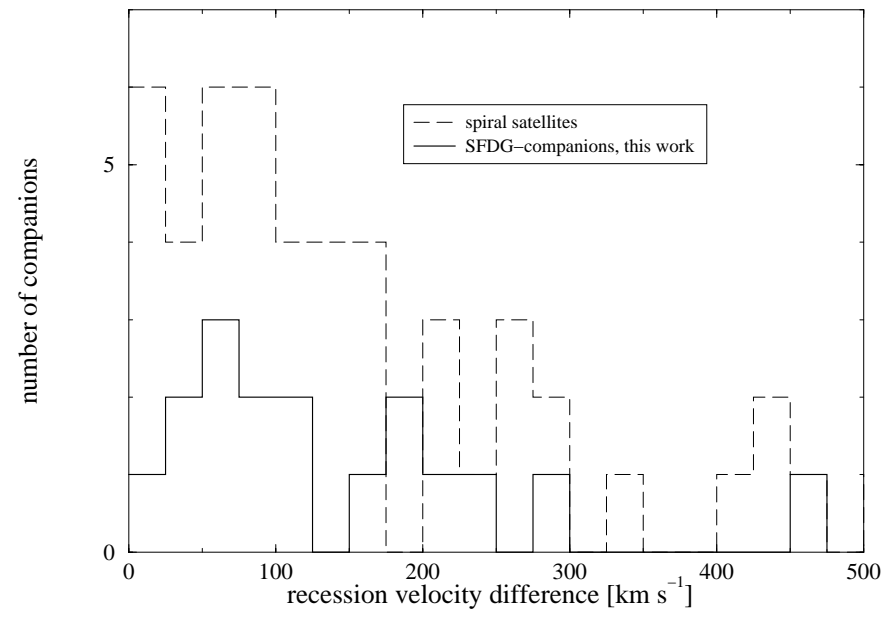

Fig. 2. Distribution of the recession velocity differences between the sample SFDGs and the putative companions. The dashed histogram is built from the spiral companions shown in Fig. 1

Table 1 (first row) shows the companion statistics obtained by the latter search criteria.

We will in the following refer to the galaxies with at least one possible companion within the limits stated above as to the non-isolated sample and to those for which no companion candidates were found as to the isolated galaxies. This is for the sake of simplicity; one has to keep in mind that the isolation criteria described above are empirical, being based on assumptions and observational clues.

\subsubsection{Companion properties; bias against faint companions}

The first row of Table 1 shows that the majority ( $\gtrsim 80 \%)$ of the possible close companions are dwarf galaxies $\left(M_{B}>\right.$ $-18 \mathrm{mag})$; this is also illustrated in Fig. 3. Their mean $B-V$ colour $(0.44 \pm 0.11 \mathrm{mag})$ is nearly equal to the average value for the sample galaxies (cf. Table 2), pointing to recent or ongoing SF activity. The differences between the $B$ luminosities of each sample galaxy and its companions are shown in Fig. 4. This distribution shows a large scatter around a median of $0.72 \mathrm{mag}$, which suggests that we tend to find primarily the brightest companion galaxies (cf. Sect. 4).

The observational bias against low-luminosity companions, i.e. against the majority of close companions of SFDGs, is also evident from Table 1 . The rough completeness limit of the NED of $\sim 15.5 \mathrm{~B}$ mag (cf. Sect. 2), translates, by the $H_{0}$ adopted herein (Sect. 1) and assuming a pure Hubble flow, to a maximum distance of $50 \mathrm{Mpc}\left(\mathrm{c} z \approx 3760 \mathrm{~km} \mathrm{~s}^{-1}\right)$ for a dwarf companion $\left(M_{B}>-18 \mathrm{mag}\right)$ to be listed in the NED. Indeed, for $c z \geq 4000 \mathrm{kms}^{-1}$, no dwarf companions were found. In the determination of the fraction of SFDGs which have a possible dwarf companion, one is therefore forced to stick to small redshifts. This is further demonstrated by the fact

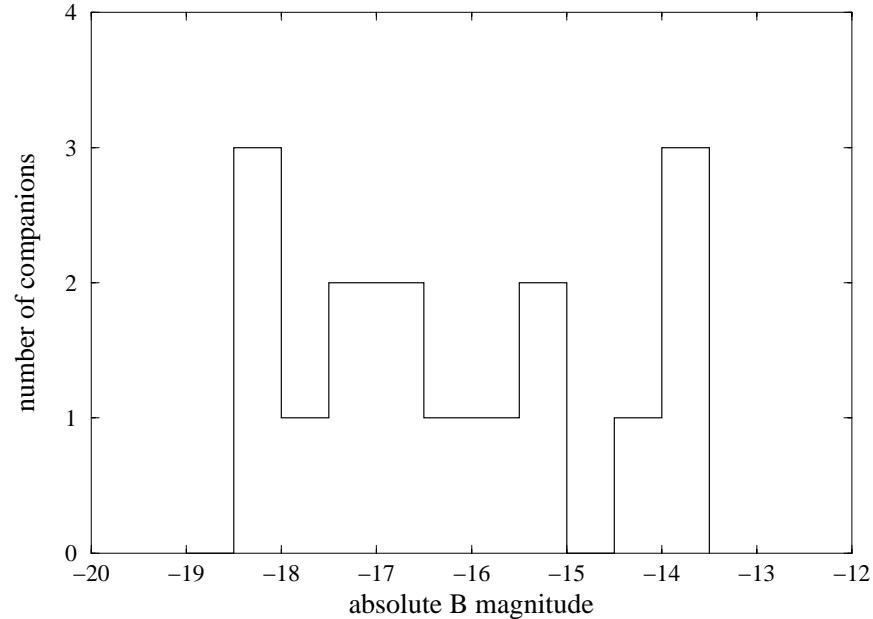

Fig. 3. Distribution of the $B$ magnitudes of the SFDG companion candidates

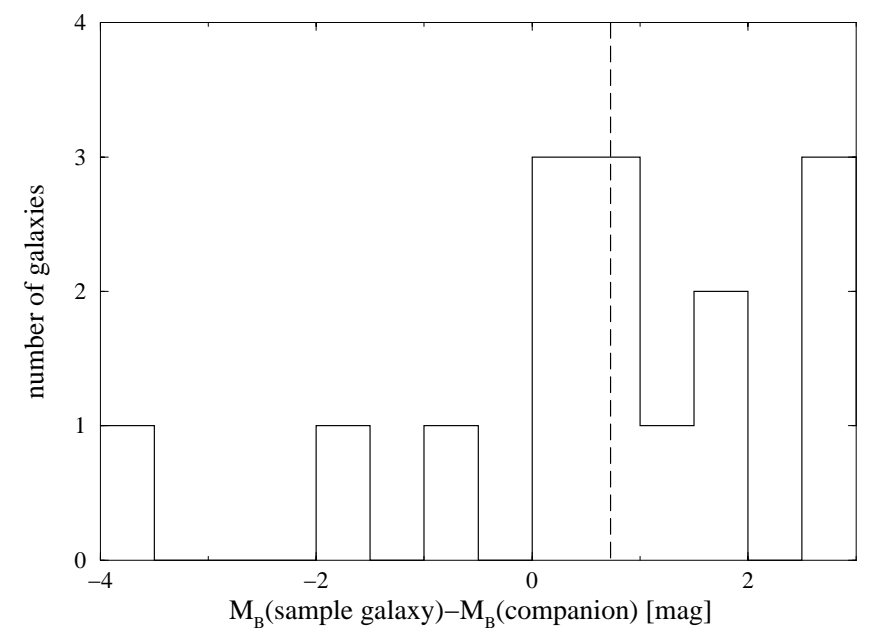

Fig. 4. Distribution of differences between the $B$ luminosity of each SFDG and its found companion candidate(s). The median value of the distribution, $0.72 \mathrm{mag}$, is marked by the dashed line

that for the subsample at $c z \leq 2000 \mathrm{~km} \mathrm{~s}^{-1}$, the fraction of non-isolated galaxies is considerably higher (31\%) than for the total sample $(16 \%)$. We will further comment on this point in Sect. 4 .

\subsection{Star formation and environment}

To assess whether the SF activity of a SFDG can be influenced by its close environment, we chose those commonly used indicators which were available for most of our sample galaxies and trace the relative strength of the SF activity on different timescales (see e.g. Schaerer 1999 for a review):

(i) The $\mathrm{H} \beta$ equivalent width $E W(\mathrm{H} \beta)$, approximately relating the flux of Lyman continuum photons, i.e. the actual or very recent $\left(\tau_{\text {burst }} \lesssim 10 \mathrm{Myr}\right)$ SFR, to the continuum flux in the $B$ band, which is usually dominated by stellar emission.

(ii) The integral $B-V$ colour of the galaxies which is an 

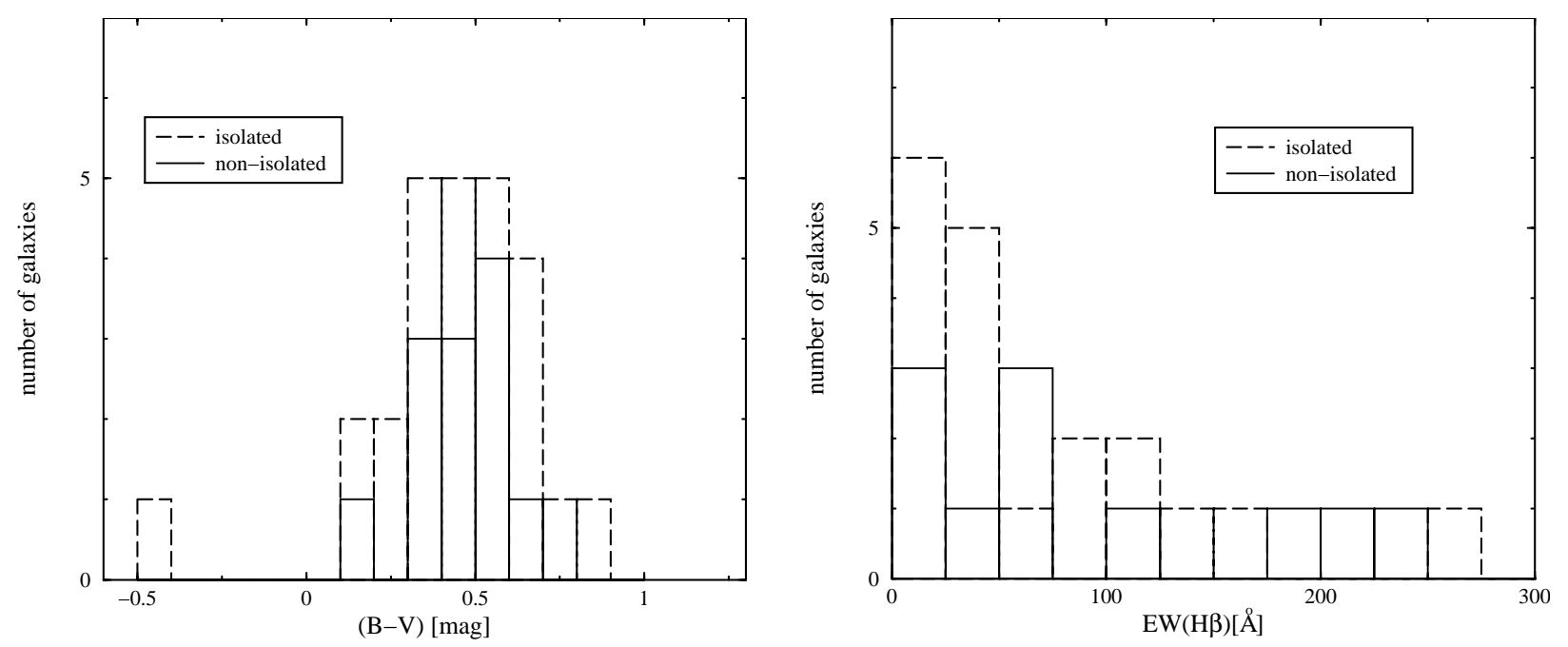

Fig. 5. Histograms of the left: $B-V$ colours and right: $\mathrm{H} \beta$ equivalent widths $E W(\mathrm{H} \beta)$ for the isolated (dashed line) and the non-isolated (solid line) subsample of SFDGs. The comparison is restricted to the sample at close distances, i.e. at redshifts $c z<2000 \mathrm{kms}^{-1}$

Table 2. Comparison of the SF activity indicators for different subsamples

\begin{tabular}{lccc}
\hline \hline SF indicator & isolated & non-isol. & K.-S. \\
$(1)$ & $(2)$ & $(3)$ & $(4)$ \\
\hline$E W(\mathrm{H} \beta)[\AA]$ & $68.4 \pm 15.1$ & $94.6 \pm 24.7$ & 0.45 \\
$\#$ data points & 19 & 11 & \\
\hline$(B-V)[\mathrm{mag}]$ & $0.43 \pm 0.07$ & $0.48 \pm 0.06$ & 0.90 \\
\# data points & 27 & 13 & \\
\hline \hline
\end{tabular}

(2) Mean of the respective SF activity indicator for the "isolated" subsample with standard deviation about the mean.

(3) Like (2), but for the "non-isolated" subsample.

(4) Result of a Kolmogorov-Smirnov test, applied to the distribution of the respective SF activity indicator for the "nonisolated" and "isolated" subsamples. The probability of the hypothesis that both distributions are drawn from the same parent distribution is shown; small values suggest that the distributions are different from each other.

indicator of the past SF activity on timescales of $\sim 10^{8} \mathrm{yr}$ (see e.g. the models by Krüger 1992 for different burst parameters). We refrain from a discussion of IRAS fluxes, originally collected and analyzed for the sample as well, as they are available for few SFDGs only, and possibly biased towards metal-rich dwarfs (see Dultzin-Hacyan et al. 1990).

In view of the bias against faint companions (Sect. 3.1.2) we stick here to the SFDGs with redshifts $c z<2000 \mathrm{~km} \mathrm{~s}^{-1}$. The abovementioned SF indicators for the isolated and non-isolated samples are compared in Fig. 5. The corresponding statistics are listed in Table 2. Column (4) lists a comparison of the sample distributions by means of Kolmogorov-Smirnov tests. Both the $E W(\mathrm{H} \beta)$ and $B-V$ colours of the isolated and nonisolated subsamples are compatible with the hypothesis that they are drawn from equal parent distributions.
Similarly, the sample means of $E W(\mathrm{H} \beta)$ and $B-V$ are compatible for the isolated and non-isolated subsamples within their respective sample standard deviations. Hence, from the available data, we do not confirm the higher mean $E W(\mathrm{H} \beta)$ of the "non-isolated" sample.

\section{Discussion}

\subsection{Frequency, distribution and properties of the companion candidates}

The present investigation reveals that a significant fraction of SFDGs possesses possible close companions which are in almost all cases dwarf galaxies. However, the selection of the nearby SFDG subsample at $c z<2000 \mathrm{~km} \mathrm{~s}^{-1}$ does not eliminate, but merely moderates, the degree of incompleteness, which is illustrated by the distribution of the $B$ magnitudes of the companion candidates (Fig. 3): unlike that expected for a complete sample, it shows no increase towards fainter luminosities. In this respect, the median of the magnitude differences between a sample galaxy and its found companion candidate(s) ( $0.7 B$ mag, Fig. 4$)$ should be interpreted as due to selection effects: the companions of SFDGs are not necessarily brighter than the SFDGs themselves, but only the brightest among the companions are listed in NED. Nevertheless, even these brightest companions qualify as dwarfs. The integrated colours of these companions are similar to those of the sample SFDGs (cf. Sect. 3.1.2), being consistent with a similar recent SF history in both sample SFDG and found companions. While this could be interpreted as a result of mutual interaction, it might again reflect the abovementioned selection effect: faint companions are most likely to be detected in emission line surveys (cf. Salzer et al. 1989b), which, of course, list preferably actively starforming galaxies.

The quantification of the true frequency of dwarf companions suffers from the principal drawback that both 
Table 3. Sample list: (1) name; (2) recession velocity in $\mathrm{km} \mathrm{s}^{-1}$, corrected for peculiar velocities introduced by Virgo Cluster perturbations (see Sect. 2.3); (3) integral $B-V$ colour; (4) $\mathrm{H} \beta$ equivalent width (in emission); (5) number of close companion candidates found in the NED within the limits described in Sect. 3.1.1

\begin{tabular}{|c|c|c|c|c|}
\hline Name & $\begin{array}{c}c z^{\text {corr. }} \\
\mathrm{km} \mathrm{s}^{-1}\end{array}$ & $\begin{array}{r}B-V \\
\text { mag }\end{array}$ & $\begin{array}{r}E W(\mathrm{H} \beta) \\
\AA\end{array}$ & \# Comp. \\
\hline (1) & (2) & (3) & (4) & (5) \\
\hline UM 306 & 4985 & 0.42 & 26.4 & 0 \\
\hline UM 92 & 6949 & 0.33 & 38.3 & 0 \\
\hline UM 323 & 1998 & 0.38 & 25.9 & 0 \\
\hline UM 330 & 5141 & 0.57 & 18.8 & 0 \\
\hline UM 334 & 4924 & 0.32 & 11.4 & 0 \\
\hline UM 335 & 4984 & 0.13 & - & 0 \\
\hline UM 336 & 5816 & 0.24 & 54.9 & 0 \\
\hline UM 345 & 5754 & 0.57 & 25.7 & 0 \\
\hline UM 351 & 7487 & 0.55 & 44.5 & 0 \\
\hline UM 369 & 5812 & 0.40 & 58.2 & 0 \\
\hline UM 371 & 5536 & 0.49 & 6.5 & 0 \\
\hline UM 372 & 1752 & 0.31 & 92.7 & 0 \\
\hline UM 374 & 5735 & 0.59 & 34.6 & 0 \\
\hline UM 379 & 8227 & 0.78 & 26.6 & 0 \\
\hline UM 151 & 4890 & 0.46 & 9.8 & 0 \\
\hline UM 382 & 3786 & 0.39 & 124.2 & 0 \\
\hline UM 396 & 6208 & 1.00 & 123.5 & 0 \\
\hline UM 404 & 3712 & 0.45 & 74.1 & 1 \\
\hline UM 406 & 11251 & 0.92 & 104.1 & 0 \\
\hline UM 408 & 3637 & 0.53 & 46.8 & 0 \\
\hline UM $410 \mathrm{~N}$ & 6960 & 0.62 & 35.4 & 1 \\
\hline UM 411 & 11748 & 0.78 & 480.5 & 0 \\
\hline UM 417 & 2846 & 0.19 & 63.6 & 0 \\
\hline UM 422 & 2017 & 0.21 & 328.6 & 0 \\
\hline UM 439 & 949 & 0.27 & 41.2 & 0 \\
\hline UM 442 & 7707 & 0.41 & 24.8 & 0 \\
\hline UM 444 & 6332 & 0.65 & 36.2 & 0 \\
\hline UM 446 & 1418 & 0.45 & 34.8 & 0 \\
\hline UM 452 & 1219 & 0.63 & 13.5 & 0 \\
\hline UM 454 & 3592 & 0.42 & 21.4 & 0 \\
\hline UM 455 & 3657 & 0.36 & 59.7 & 0 \\
\hline UM 456 & 1518 & 0.34 & 42.7 & 0 \\
\hline UM 461 & 735 & 0.43 & 241.2 & 1 \\
\hline UM 462 & 855 & 0.44 & 72.6 & 1 \\
\hline UM 463 & 1131 & 0.45 & 98.5 & 0 \\
\hline UM 465 & 1155 & 0.59 & 15.7 & 1 \\
\hline UM 471 & 10185 & 0.76 & 41.8 & 0 \\
\hline UM 483 & 2099 & 0.92 & 27.7 & 0 \\
\hline UM 487 & 14419 & 0.50 & 6.6 & 0 \\
\hline UM 490 & 5376 & -0.15 & 113.0 & 0 \\
\hline UM 491 & 1753 & 0.42 & 14.6 & 0 \\
\hline UM 495 & 7477 & 0.52 & 47.7 & 0 \\
\hline UM 496 & 11479 & 0.73 & 110.9 & 0 \\
\hline UM 500 & 1805 & 0.15 & 117.8 & 1 \\
\hline UM 501 & 1745 & 0.39 & 184.5 & 1 \\
\hline UM 504 & 1867 & 0.50 & 17.9 & 0 \\
\hline UM 507 & 6094 & 0.58 & 94.2 & 0 \\
\hline UM 512 & 4488 & 0.51 & 13.8 & 0 \\
\hline UM 513 & 3457 & 0.82 & 7.3 & 0 \\
\hline
\end{tabular}

\begin{tabular}{|c|c|c|c|c|}
\hline Name & $\begin{array}{r}c z^{\text {corr. }} \\
\mathrm{km} \mathrm{s}^{-1} \\
(2)\end{array}$ & $\begin{array}{r}B-V \\
\text { mag } \\
(3)\end{array}$ & $\begin{array}{r}E W(\mathrm{H} \beta) \\
\AA \\
(4)\end{array}$ & $\begin{array}{r}\text { \# Comp. } \\
\text { (5) }\end{array}$ \\
\hline UM 523 & 1620 & 0.51 & 16.9 & 1 \\
\hline UM 533 & 737 & 0.35 & 206.5 & 1 \\
\hline UM 538 & 855 & 0.56 & 41.2 & 0 \\
\hline UM 539 & 6065 & 0.60 & 56.5 & 0 \\
\hline UM 549 & 5642 & 0.36 & 15.5 & 0 \\
\hline UM 552 & 7190 & 0.48 & 20.9 & 0 \\
\hline UM 559 & 1037 & 0.18 & 261.7 & 0 \\
\hline UM 562 & 5290 & 0.91 & 199.5 & 0 \\
\hline UM 564 & 13668 & 0.86 & 101.1 & 0 \\
\hline UM 570 & 6543 & 1.18 & 199.2 & 0 \\
\hline UM 588 & 3581 & 0.47 & 18.6 & 1 \\
\hline UM 591 & 15642 & 0.61 & 38.6 & 0 \\
\hline UM 597 & 6441 & 0.46 & 22.7 & 0 \\
\hline UM 605 & 4956 & 0.59 & 6.1 & 0 \\
\hline UM 612 & 4414 & 0.54 & 19.1 & 0 \\
\hline UM 618 & 4188 & 0.39 & 39.5 & 0 \\
\hline UM 619 & 4455 & 0.63 & 10.4 & 0 \\
\hline UM 626 & 3335 & 0.57 & 5.1 & 0 \\
\hline UM 628 & 7052 & 0.15 & 32.3 & 0 \\
\hline UM 635 & 7192 & 0.47 & 25.9 & 0 \\
\hline UM 648 & 9715 & 0.78 & 4.4 & 0 \\
\hline UM 649 & 7554 & 0.58 & 81.7 & 0 \\
\hline UGC 47 & 873 & 0.34 & - & 1 \\
\hline TOL 0127-397 & 4797 & 0.59 & 38.0 & 0 \\
\hline DDO 19 & 735 & 1.21 & - & 0 \\
\hline MKN 370 & 840 & 0.63 & 10.0 & 0 \\
\hline MKN 600 & 945 & 0.46 & - & 0 \\
\hline UGC 2432 & 764 & 0.89 & - & 0 \\
\hline UGC 2482 & 2646 & 0.61 & 一 & 0 \\
\hline UGC 2809 & 1239 & 0.49 & - & 0 \\
\hline c0341-4045 & 4497 & 0.46 & 一 & 0 \\
\hline DDO 34 & 615 & -0.40 & - & 0 \\
\hline MKN 5 & 792 & 0.50 & 150.0 & 0 \\
\hline UGC 3516 & 1287 & 0.65 & - & 0 \\
\hline UGC 3658 & 1267 & 0.29 & 一 & 0 \\
\hline MKN 86 & 675 & 0.66 & 6.2 & 2 \\
\hline I Zw 18 & 1072 & 0.19 & 69.0 & 0 \\
\hline MKN 36 & 517 & 0.46 & 70.0 & 1 \\
\hline VII ZW 403 & 105 & - & - & 0 \\
\hline NGC 4670 & 825 & 0.39 & 一 & 0 \\
\hline MKN 209 & 352 & 0.53 & 60.3 & 2 \\
\hline II Zw 70 & 1732 & 0.52 & 49.0 & 1 \\
\hline II Zw 71 & 1785 & 1.23 & - & 1 \\
\hline PHL 293 B & 1762 & 0.77 & 110.0 & 0 \\
\hline MKN 324 & 1635 & 0.60 & 3.8 & 0 \\
\hline MKN 67 & 1680 & 0.31 & 105.0 & 0 \\
\hline MKN 169 & 1777 & 0.51 & 21.9 & 0 \\
\hline I Zw 123 & 1125 & 0.60 & 144.5 & 0 \\
\hline HARO 38 & 1327 & - & - & 0 \\
\hline
\end{tabular}


the low-luminosity end of the galaxy LF and the frequency of $\mathrm{H}$ I companions without any optical counterpart (Taylor et al. 1994, 1995, 1996) are not yet wellconstrained. Therefore, even a uniform and well-defined homogeneous completeness limit for the NED would to date not allow any meaningful extrapolation. Instead, we emphasize that the value of $\approx 30 \%$ inferred in Sect. 3.1 .2 must be considered a lower limit to the true fraction of SFDGs with close optical or purely gaseous (dwarf) companions within $\Delta c z<500 \mathrm{~km} \mathrm{~s}^{-1}$ and $s_{\mathrm{p}}<0.1 \mathrm{Mpc}$. It must be noted that this work and the one by Pustilnik et al. (2001), performed independently and using different approaches and data, find almost equal frequencies of lowluminosity companions of SFDGs/BCDs. Furthermore, Pustilnik et al. (2001) also report evidence for a considerable number of even fainter, but yet uncatalogued companions. Altogether, this lends further support to the results by L96, who attributed the apparent extreme isolation of some BCDs to an observational bias against distant, intrinsically faint companions.

The small redshift differences between SFDGs and companions, the distribution of which rises towards smaller values, are comparable to what is found for binary galaxies (Schneider \& Salpeter 1992). In particular, a similar behaviour is reported for dwarf satellites of field spirals (Zaritsky et al. 1997). This is illustrated in Figs. 1 and 2, where we plot our results of a near-object search (not restricted to dwarf galaxies) in the NED around field spirals from Kennicutt \& Kent (1983). Analogous to spiral companions, there is no evident correlation between $\Delta c z$ and $s_{\mathrm{p}}$ for the SFDG companions. A plausible explanation of this issue was offered in the framework of a CDM scenario, taking into account a number of further dynamical arguments, by Zaritsky et al. (1997). They consider the spiral satellites to be associated with an extended massive DM halo of the mother galaxy, which strongly influences the system's dynamics, so that $\Delta c z$ is widely independent of $s_{\mathrm{p}}$. Figure 1 suggests that $\Delta c z$ and $s_{\mathrm{p}}$ are also uncorrelated for the SFDG-companion systems. If this can be corroborated by a larger dataset, then a hypothesis worth investigating for compatibility with CMD models is that SFDGs and their companions share common DM haloes of similar mass and structure.

At least, the data strongly suggest some physical association between SFDGs and their companions. In combination with our considerations on the frequency of dwarf companions, this leads to the assumption that SFDGs are frequently associated with systems of two or more dwarf galaxies. This is reminiscent of the dwarf galaxy groups L96 found in voids, and may offer important clues to dwarf galaxy formation.

\subsection{Close dwarf companions as starburst triggers?}

The similarity of the SF tracers for the isolated and nonisolated subsamples may suggest that the observed starburst activity is regulated by solely internal processes.
This hypothesis can, on the other hand, not be proven, and neither can an influence of the presence or absence of companions be disproven. Indeed, this latter hypothesis may receive some support from the marginal trend in $E W(\mathrm{H} \beta)$ that the data shows. The large scatter of the analyzed SF diagnostics is not surprising, for a number of reasons:

(i) The severe incompleteness of the available data aggravates the separation of galaxies with and without close companions;

(ii) Balmer emission lines, i.e. Lyman continuum tracers, correspond to short-term variations in the SFR. The latter are particularly strong in SFDGs, where SF generally occurs in short bursts of $\lesssim 10^{7}$ yr (Elmegreen et al. 1996). Considerable variations with time also occur for the $B-V$ colours after the onset of a starburst (see e.g. Krüger 1992). Whatever triggers a starburst, the time window during which strongly enhanced SF indicators are observable is narrow. In addition, such SFR tracers also depend on evolutionary parameters - metallicity, age and stellar continuum contribution - of the SFDG itself.

The hypothesis of interaction-induced starburst activity in SFDGs might be more reliably assessed when a large sample of SFDGs with sufficiently deeply observed environments is available. However, the abovementioned intrinsic sources of scatter certainly limit the usefulness of a comparison that is restricted to integral SFR tracers.

This research emphasizes the need for a systematic effort on the part of theory to advance the understanding of conditions required for inducing and sustaining starburst activity in dwarf galaxies, with particular emphasis on dwarf galaxy interactions.

\section{Summary and conclusions}

We have searched the close environment of a sample of 98 star-forming dwarf galaxies (SFDGs) in field and lowdensity environments, taken from different catalogues, for possible companion galaxies. To supplement previous work, which had mostly dealt with luminous companions, a subsample of nearby SFDGs was chosen to moderate the effect of observational bias against low luminosity companions. Distances were obtained from the recession velocity of the sample galaxies assuming a pure Hubble flow and have been corrected for Virgocentric infall. Using the Nasa Extragalactic Database (NED) as a search catalogue, objects with a projected linear separation of $<100 \mathrm{kpc}$ and a recession velocity difference $<2000 \mathrm{~km} \mathrm{~s}^{-1}$ were catalogued as possible companions of a SFDG. Most of them differ in their recession velocity by $<500 \mathrm{kms}^{-1}$ from the sample SFDGs, which, along with a number of other considerations, led us to adopt this value as a limit to identify possible companions. For both SFDGs and companion candidates, spectrophotometric data were compiled from the NED and a number of literature sources.

We studied the frequency, redshift difference distribution, and photometric properties of companion candidates, as well as their possible influence on the star formation 
(SF) activity of the sample SFDGs. Our results can be summarized as follows:

(i) A substantial fraction of SFDGs possess companion galaxies within its close environment. The overwhelming majority of these companions are dwarfs $\left(M_{B}>-18\right)$, which renders their detection at large distances difficult. The fraction of SFDGs for which we detect dwarf companion candidates, $\approx 30 \%$, must be considered a lower limit to the true value, given the increasing incompleteness of the available data set for systems fainter than $m_{B} \sim 15.5 \mathrm{mag}$. A meaningful extrapolation to fainter magnitudes is precluded by the poorly constrained frequency of H I companions with no optical counterpart, and by the uncertain faint end of the galaxy luminosity function;

(ii) The recession velocity differences between SFDGs and identified companions amount typically to $\$ 250 \mathrm{~km} \mathrm{~s}^{-1}$. Their frequency rises towards lower differences. This is similarly reported for dwarf companions of spirals, and suggests that the SFDG companions are physically associated with the sample galaxies;

(iii) Both the $B-V$ colours and the $\mathrm{H} \beta$ equivalent widths appear compatible for objects with and without a possible perturbor. The significance level of the data is too low to prove the hypothesis of a solely internal regulation of the SF activity, whereas external influence cannot be disproven either. The considerable intrinsic scatter inherent to statistical studies based on the abovementioned SF diagnostics, together with the insufficiently complete companion search databases, blurs the picture.

The identification of companions in the close environment of SFDGs readdresses the question of whether gravitational interactions are partly responsible for the onset of SF activity in gas-rich dwarfs. Whereas interactioninduced SF activity was dismissed for most SFDGs due to the absence of luminous companions, the presence of faint dwarfs in the close vicinity of SFDGs provides again support to this hypothesis. While deep radio and spectrophotometric surveys may yield more conclusive answers, it will equally be important to theoretically model the effects of interactions on dwarf galaxies.

Acknowledgements. KGN gratefully acknowledges financial support from the German Research Foundation (DFG) grant FR325/50-1, and from the IAC Summer Research Programme 1998. This study was partly financed by the Spanish DGES (Dirección General de Enseñanza Superior) (grant PB97-0158). PP received support from Deutsche Agentur für Raumfahrtangelegenheiten (DARA) GmbH grant 50 OR 9907 7. This Research has made use of the NASA/IPAC Extragalactic Database (NED) which is operated by the Jet Propulsion Laboratory, CALTECH, under contract with the National Aeronautic and Space Administration. We have made use of the Lyon-Meudon Extragalactic Database (LEDA) supplied by the LEDA team at the CRAL-Observatoire de Lyon (France). We thank S. A. Pustilnik and A. Kniazev for fruitful discussions and helpful comments on this paper. KGN wishes to thank L. M. Cairós, J. Iglesias, J. Vílchez and the IAC staff for their hospitality. We thank the referee, Dr. Metcalfe, for his helpful comments.

\section{References}

Cairós, L. M., \& Vílchez, J. M. 1998, in The Magellanic Clouds and other Dwarf Galaxies, ed. T. Richtler, \& J. M. Braun (Shaker Verlag), 235

Cairós, L. M., Vílchez, J. M., González-Pérez, J. N., et al. 2000, ApJ, in press

Campos-Aguilar, A., \& Moles, M. 1991, A\&A, 241, 358

Campos-Aguilar, A., Moles, M., \& Masegosa, J. 1993, AJ, 106, 1784

Davies, J. I., \& Phillipps, S. 1988, MNRAS, 23, 553

Doublier, V., Caulet, A., \& Comte, G. 1999 A\&AS, 138, 213

Dultzin-Hacyan, D., Masegosa, J., \& Moles, M. 1990, A\&A, 238, 28

Elmegreen, B. G., Elmegreen, D. M., Salzer, J. J., \& Mann, H. 1996, ApJ, 467, 579

French, H. B. 1980, ApJ, 240, 41

Garcia, A. M. 1993, A\&AS, 100, 47

Gerola, H., Seiden, P., \& Schulmann, L. 1980, ApJ, 242, 517

Grogin, N. A., \& Geller, M. J. 2000, AJ, 119, 32

Guzmán, R., Jangren, A., Koo, D. C., Bershady, M. A., \& Simard, L. 1998, ApJ, 495, L13

Hashimoto, Y., Oemler, A., Lin, H., \& Tucker, D. L. 1998, ApJ, 499, 589

Hensler, G., Rieschick, A., \& Köppen, J. 1999, in The Evolution of Galaxies on Cosmological Timescales, ed. J. Beckman, \& T. Mahoney, Ap\&SS, in press [astro-ph/9908242]

Kennicutt, R. C., \& Kent, S. M. 1983, AJ, 88, 1094

Kennicutt, R. C., Roettiger, K. A., Keel, W. C., van der Hulst, J. M., \& Hummel, E. 1987, AJ, 93, 1011

Kraan-Korteweg, R. C. 1986, A\&AS, 66, 255

Krüger, H. 1992, Ph.D. Thesis, Universitäts-Sternwarte Göttingen

Laurikainen, E., Salo, H., \& Aparicio, A. 1998, A\&AS, 129, 517

Lee, J. C., Salzer, J. J., Law, D. A., \& Rosenberg, J. L. 2000, ApJ, 536, 606

Lindner, U., Einasto, M., Einasto, J., et al. 1996, A\&A, 314, 1 (L96)

Loveday, J., Tresse, L., \& Maddox, S. 1999, MNRAS, 310, 281

Marinoni, C., Monaco, P., Giuricin, G., \& Costantini, B. 1998, ApJ, 505, 484

Marlowe, A. T., Meurer, G. R., \& Heckman, T. M. 1999, ApJ, 522,183

McAlpine, G. M., Lewis, D. W., \& Smith, S. B. 1977a, ApJS, 35,203

McAlpine, G. M., Smith, S. B., \& Lewis, D. W. 1977b, ApJS, 34,95

McAlpine, G. M., Smith, S. B., \& Lewis, D. W. 1977c, ApJS, 35,197

McAlpine, G. M., \& Lewis, D. W. 1978, ApJS, 36, 587

McAlpine, G. M., \& Williams, G. A. 1981, ApJS, 36, 587

Méndez, D. I., Cairós, L. M., Esteban, C., \& Vílchez, J. M. 1999, AJ, 117, 1688

Méndez, D. I., \& Esteban, C. 1999, AJ, 118, 2723

Noeske, K. G. 1999, Diploma Thesis, Universitäts-Sternwarte Göttingen

Noeske, K. G., Cairós, L. M., Papaderos, P., Vílchez, J. M., \& Fricke, K. J. 1998, in The Evolution of Galaxies on Cosmological Timescales, ed. J. Beckman, \& T. Mahoney, Ap\&SS, in press

Noeske, K. G., Papaderos, P., Fricke, K. J., \& Thuan, T. X. 1998, in The Magellanic Clouds and other Dwarf Galaxies, ed. T. Richtler, \& J. M. Braun (Shaker Verlag), 279 
Odewahn, S. C. 1994, AJ, 107, 1320

Papaderos, P., Loose, H.-H., Fricke, K. J., \& Thuan, T. X. 1996, A\&A, 314, 59

Pilyugin, L. 2000, A\&A, 354, 874

Popescu, C. C., Hopp, U., \& Rosa, R. 1999, A\&A, 350, 414

Pustilnik, S. A., Kniazev, A. Y., \& Ugryumov, A. V. 1997, IAUJD, 2E, 60

Pustilnik, S. A., Kniazev, A. Y., Lipovetsky, V. A., \& Ugryumov, A. V. 2001, A\&A, submitted

Pustilnik, S. A., Ugryumov, A. V., Lipovetsky, V. A., Thuan, T. X., \& Guseva, N. 1995, ApJ, 443, 499

Rosenberg, J. L., \& Salzer, J. J. 1994, AJ, 108, 1557

Salzer, J. J. 1989, ApJ, 347, 152

Salzer, J. J., McAlpine, G. M., \& Boroson, T. A. 1989a, ApJS, 70,447

Salzer, J. J., McAlpine, G. M., \& Boroson, T. A. 1989b, ApJS, 70,479

Salzer, J. J., \& Norton, S. A. 1998, IAUC, 171

Savage, B. D., \& Mathis, J. S. 1979, ARA\&A, 17, 73

Schaerer, D. 1999, in Building the Galaxies: from the Primordial Universe to the Present, XIXth Moriond astrophysics meeting, ed. F. Hammer, et al. (World Scientific), 389

Schneider, S. E., \& Salpeter, E. E. 1992, ApJ, 385, 32

Shull, J. M., Stocke, J. T., \& Penton, S. 1996, AJ, 111, 72

Taylor, C. L., Brinks, E., Podge, R. W., \& Skillman, E. D. 1994, AJ, 107, 971
Taylor, C. L., Brinks, E., Grashius, R. M., \& Skillman, E. D. 1995, ApJS, 102, 189

Taylor, C. L., Thomas, D. L., Brinks, E., \& Skillman, E. D. 1996, ApJS, 107, 143

Telles, E., \& Terlevich, R. 1995, MNRAS, 275, 1 (TT95)

Telles, E., \& Maddox, S. 1999, MNRAS, 311, 307

Terlevich, R., Melnick, J., Masegosa, J., Moles, M., \& Copetti, M. V. F. 1991, A\&AS, 91, 885 (SCHG)

Thuan, T. X. 1985, ApJ, 299, 881

Thuan, T. X. 1991, in Massive Stars in Starbursts, ed. C. Leitherer, N. R. Walborn, T. M. Heckman, \& C. A. Norman (Cambridge University Press), 183

Thuan, T. X., \& Martin, G. 1981, ApJ, 247, 823

Tully, R. B. 1988, Nearby Galaxies Catalog (Cambridge University Press)

Tully, R. B., \& Shaya, E. J. 1984, ApJ, 281, 31

Vennik, J., Hopp, U., \& Popescu, C. C. 2000, A\&AS, 142, 399

Vílchez, J. M. 1995, AJ, 110, 1090

Vílchez, J. M. 1997, RMxAA (Serie de Conferencias), 6, 30

Walter, F., Brinks, E., Duric, N., \& Klein, U. 1997, AJ, 113, 2031

Wilcots, E. M., Lehman, C., \& Miller, B. 1996, AJ, 111, 1575

Zaritsky, D., Smith, R., Frenk, C., \& White, S. D. M. 1997, ApJ, 478, 39

Zwicky, F. 1961, Catalogue of Galaxies and Clusters of Galaxies (CGCG) 